\title{
REFERENCES
}

1. R. P. Boas, $A$ theorem on analytic functions of a real variable, Bull. Amer. Math. Soc. vol. 41 (1935) pp. 233-236.

2. Alberto Calderon and Allen Devinatz, Sur certaines courbes dans l'espace de Hilbert, C. R. Acad. Sci. Paris vol. 241 (1955) pp. 539-541.

3. - Sur certaines courbes d courbure constante dans l'espace de Hilbert, C. R. Acad. Sci. Paris vol. 241 (1955) pp. 586-587.

4. Carl-Gustave Esseen, Fourier analysis of distribution functions, Acta Math. vol. 77 (1945) pp. 1-125.

UNIVERSITY OF CONNECTICUT AND

WASHINGTON UNIVERSITY

\section{ON A SERIES OF RAINVILLE INVOLVING LEGENDRE POLYNOMIALS}

B. R. BHONSLE

1. The object of this paper is to obtain some relations involving Legendre polynomials with the help of a series given by E. D. Rainville. The results are believed to be new.

2. We start with the series given by E. D. Rainville

$$
P_{n}(\cos \alpha)=\left(\frac{\sin \alpha}{\sin \beta}\right)^{n} \sum_{k=0}^{n} c_{n, k}\left[\frac{\sin (\beta-\alpha)}{\sin \alpha}\right]^{n-k} P_{k}(\cos \beta) .
$$

Putting $\beta=2 \alpha$ and $\cos 2 \alpha=x$, we get

$$
2^{n / 2}(1+x)^{n / 2} P_{n}\left(\left(\frac{1+x}{2}\right)^{1 / 2}\right)=\sum_{k=0}^{n} c_{n, k} P_{k}(x) .
$$

From (2.2) and the orthogonal property

$$
\begin{aligned}
\int_{-1}^{1}(1+x)^{n / 2} P_{r}(x) P_{n}\left(\left(\frac{1+x}{2}\right)^{1 / 2}\right) d x & \\
=\frac{c_{n, \gamma}}{2^{n / 2-1}(2 \gamma+1)}, & 0 \leqq \gamma \leqq n, \\
=0, & r>n .
\end{aligned}
$$

Using (2.3) with Adams' expansion (Modern analysis, p. 331) for

Received by the editors October 2, 1955 and, in revised form, March 26, 1956. 
$P_{p}(x) P_{q}(x)$, where $p$ and $q$ are positive integers and $q \leqq p$,

$$
\begin{aligned}
& \int_{-1}^{1}(1+x)^{n / 2} P_{n}\left(\left(\frac{1+x}{2}\right)^{1 / 2}\right) P_{p}(x) P_{q}(x) d x \\
& \quad=\frac{1}{2^{n / 2-1}} \sum_{\gamma=0}^{q} \frac{A_{p-\gamma} A_{\gamma} A_{q-\gamma} C_{n, p+q-2 \gamma}}{A_{p+q-\gamma}(2 p+2 q-2 \gamma+1)}=\frac{(1 / 2)_{p}(1 / 2)_{q} n !}{(1 / 2)_{p+q+1} p ! q ! 2^{n / 2}} \\
& \times{ }_{6} F_{5}\left[\begin{array}{l}
-q,-p, \frac{1}{2}-p-q,-\frac{1}{2}(p+q),-\frac{1}{2}(p+q-1), \frac{1}{2} ; \\
-q-p, \frac{1}{2}-p, \frac{1}{2}-q, \frac{1}{2}(n+1-p-q), \frac{1}{2}(n+2-p-q) ;
\end{array}\right]
\end{aligned}
$$

where

$$
\begin{aligned}
A_{\gamma} & =\frac{1 \cdot 3 \cdot 5 \cdots(2 r-1)}{\gamma !}, \\
(a)_{\gamma} & =a(a+1) \cdots(a+r-1), \quad(a)_{0}=1,
\end{aligned}
$$

and $n \geqq(p+q)$.

Also when $s=p_{1}+p_{2}+\cdots+p_{r}$, following the method of Dr. N. G. Shabde (1945) we obtain,

$$
\begin{aligned}
\int_{0}^{1} x^{s+1} P_{p_{1}}(x) P_{p_{2}}(x) \cdots P_{p_{\gamma}}(x) P_{s} & \left(2 x^{2}-1\right) d x \\
& =\frac{(s !)^{2}}{2^{s+1}(2 s+1) !} \prod_{t=1}^{\gamma} \frac{\left(2 p_{t}\right) !}{p_{t} ! p_{t} !} .
\end{aligned}
$$

With Gormley's result,

$$
x^{m}=\sum_{s=0}^{[m / 2]} \frac{(2 m-4 s+1) m ! \Gamma(1 / 2)}{2^{m+1} s ! \Gamma(m-s+3 / 2)} P_{m-2 s}(x)
$$

we find that,

$$
\begin{aligned}
\int_{-1}^{1} x^{m}(1+x)^{\bullet / 2} P_{p_{1}} & \left(\left(\frac{1+x}{2}\right)^{1 / 2}\right) \cdots P_{p_{\gamma}}\left(\left(\frac{1+x}{2}\right)^{1 / 2}\right) \\
& \times P_{p_{\gamma+1}}(x) \cdots P_{p_{\gamma+n}}(x) P_{l}(x) d x \\
& =\frac{m !(l !)^{2} \Gamma(1 / 2)(2 m+1)}{2^{m+s / 2} \Gamma(m+3 / 2)(2 l+1) !} \prod_{t=0}^{\gamma+n} \frac{\left(2 p_{t}\right) !}{p_{t} ! p_{t} !}
\end{aligned}
$$

where

$$
p_{1}+p_{2}+\cdots+p_{\gamma}=s, \quad p_{0}=m
$$

and 


$$
s+m+p_{\gamma+1}+\cdots+p_{\gamma+n}=l .
$$

Differentiating both sides of (2.2) r-times and using Grosswald's formula (1950),

$$
\begin{aligned}
& {\left[\frac{d^{\gamma}}{d x^{\gamma}}\left\{(1+x)^{n / 2} P_{n}\left(\left(\frac{1+x}{2}\right)^{1 / 2}\right)\right\}\right]_{x=1}} \\
& \quad=\sum_{k=\gamma}^{n} c_{n, k} \frac{(k+\gamma) !}{2^{n / 2+\gamma} \gamma !(k-\gamma) !} \\
& =2^{\gamma-n / 2}\left(\frac{1}{2}\right)_{\gamma} c_{n, \gamma 2} F_{1}\left[\begin{array}{lll}
-n+\gamma, & 1+2 \gamma ; & -1 \\
1+\gamma ; &
\end{array}\right] .
\end{aligned}
$$

Using Neumann's formula with (2.2) we get,

$$
\int_{-1}^{1} \frac{(1+x)^{n / 2} P_{n}\left(\left(\frac{1+x}{2}\right)^{1 / 2}\right)}{y-x} d x=2^{1-n / 2} \sum_{k=0}^{n} c_{n, k} Q_{k}(y)
$$

where $y>1$.

Also (Higher transcendental functions, p. 171, result (23))

$$
\int_{0}^{1} x^{\sigma}(1+x)^{n / 2} P_{n}\left(\left(\frac{1+x}{2}\right)^{1 / 2}\right) F_{p}\left(\begin{array}{l}
\alpha_{1}, \cdots, \alpha_{p} ; \\
\beta_{1}, \cdots, \beta_{q} ;
\end{array} \pm x^{2} t^{2}\right) d x
$$

$$
\begin{aligned}
& =\sum_{k=0}^{n} \frac{c_{n, k} \Gamma(1+\sigma / 2) \Gamma(1 / 2+\sigma / 2)}{2^{1+n / 2} \Gamma(1+\sigma / 2-k / 2) \Gamma(3 / 2+\sigma / 2+k / 2)} \\
& \times_{p+2} F_{q+2}\left(\begin{array}{l}
\alpha_{1}, \cdots, \alpha_{p}, 1+\sigma / 2,1 / 2+\sigma / 2 ; \\
\beta_{1}, \cdots, \beta_{q}, 1+\sigma / 2-k / 2,3 / 2+\sigma / 2+k / 2 ;
\end{array} \pm t^{2}\right)
\end{aligned}
$$

$|t|<1$ when $p=q+1$, and the hypergeometric series should be terminating when $p>q+1$.

We can get many particular cases of the result (2.9).

We have due to Fred Brafman (1951, p. 944) the following generating function for Legendre Polynomials $P_{n}(x)$

$$
\begin{aligned}
& { }_{2} F_{1}\left[\begin{array}{cc}
a, 1-a ; & \frac{(1-t-\rho)}{2} \\
1 ; &
\end{array}\right] \times{ }_{2} F_{1}\left[\begin{array}{cc}
a, 1-a ; & \frac{(1+t-\rho)}{2} \\
1 ; &
\end{array}\right] \\
& =\sum_{n=0}^{\infty} \frac{(a)_{n}(1-a)_{n}}{n ! n !} P_{n}(x) t^{n}
\end{aligned}
$$

with $\rho=\left(1-2 x t+t^{2}\right)^{1 / 2}$. 
The series in (2.10) is absolutely and uniformly convergent for $x$, when $|t|<1$, and therefore,

$$
\begin{aligned}
& \int_{-1}^{1}(1+x)^{n / 2}{ }_{2} F_{1}\left[\begin{array}{c}
a, 1-a ; \\
1 ;
\end{array}\right]
\end{aligned}
$$

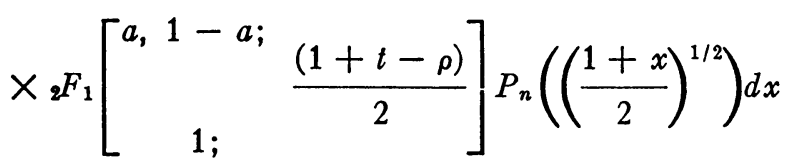

$$
\begin{aligned}
& =\sum_{k=0}^{n} \frac{c_{n, k}(a)_{k}(1-a)_{k}}{2^{n / 2-1} k ! k !(2 k+1)} t^{k} \\
& =2^{1-n / 2} F_{3}\left[\begin{array}{ccc}
-n, & a, 1-a, 1 / 2 ; \\
1, & 1, & 3 / 2 ;
\end{array}\right]
\end{aligned}
$$

with

$$
\left.\rho=1-2 x t+t^{2}\right)^{1 / 2}
$$

When in (2.11) $a=-m$, where $m$ is a positive integer we obtain,

$$
\begin{aligned}
\int_{-1}^{1}(1+x)^{n / 2} P_{m}(t+\rho) P_{m}(t-\rho) P_{n}\left(\left(\frac{1+x}{2}\right)^{1 / 2}\right) d x \\
=\sum_{k=0}^{n} \frac{c_{n, k}(-m)_{k}(m+1)_{k}}{2^{n / 2-1} k ! k !(2 k+1)} t^{k} \\
=2^{1-n / 2} F_{8}\left[\begin{array}{cc}
-n,-m, 1+m .1 / 2 ; & 1,-t] \\
1, & 1,3 ;
\end{array}\right]
\end{aligned}
$$

with

$$
\rho=\left(1-2 x t+t^{2}\right)^{1 / 2} .
$$

Again the series (Fred Brafman, 1951, p. 946),

$$
(1-x t)^{-b}{ }_{2} F_{1}\left[\begin{array}{cc}
b / 2, & (b+1) / 2 ; \\
1 ; & \frac{t^{2}\left(x^{2}-1\right)}{(1-x t)^{2}}
\end{array}\right]=\sum_{n=0}^{\infty} \frac{(b)_{n} P_{n}(x)}{n !} t^{n}
$$

is uniformly and absolutely convergent, when $|t|<1$, therefore, 
$(2.14)$

$$
\begin{aligned}
& \int_{-1}^{1}(1+x)^{n / 2}(1-x t)^{-b} \\
& \times{ }_{2} F_{1}\left[\begin{array}{cc}
b / 2,(b+1) / 2 ; & \frac{t^{2}\left(x^{2}-1\right)}{(1-x t)^{2}}
\end{array}\right] P_{n}\left(\left(\frac{1+x}{2}\right)^{1 / 2}\right) d x \\
& =\sum_{k=0}^{n} \frac{c_{n, k}(b)_{k}}{2^{n / 2-1} k !(2 k+1)} t^{k}=2^{1-n / 2}{ }_{3} F_{2}\left[\begin{array}{r}
-n, b, 1 / 2 ; \\
1,3 / 2 ;
\end{array}-t\right] \text {. }
\end{aligned}
$$

3. In (2.2) putting $((1+x) / 2)^{1 / 2}=y$, we get

$$
2^{n} y^{n} P_{n}(y)=\sum_{k=0}^{n}(-1)^{k} c_{n, k} P_{k}\left(1-2 y^{2}\right) .
$$

We have also (Mitra)

$$
\int_{0}^{1} P_{n}\left(1-2 y^{2}\right) J_{0}(y x) y d y=\frac{1}{x} J_{2 n+1}(x) .
$$

Using (3.2) with (3.1) we get,

$$
\int_{0}^{1} y^{n+1} J_{0}(y x) P_{n}(y) d y=\frac{1}{2^{n} x} \sum_{k=0}^{n}(-1)^{k} c_{n, k} J_{2 k+1}(x) .
$$

I am extremely thankful to Dr. N. G. Shabde for suggesting the problem to me. My thanks are also due to him for his help and guidance during the preparation of the paper and to Principal S. P. Chakravarti for the encouragement and facilities he gave to me. I am also highly grateful to the referee for putting the results (2.4), (2.7), (2.11), (2.12) and (2.14) in hypergeometric form, and giving other useful suggestions.

\section{REFERENCES}

1. Fred Brafman, Proc. Amer. Math. Soc. vol. 2 (1951) pp. 942-949.

2. A. Erdélyi, Higher transcendental functions, 1953.

3. P. G. Gormley, J. London Math. Soc. vol. 9 pp. 149-152.

4. E. Grosswald, Proc. Amer. Math. Soc. vol. 1 (1950) p. 553.

5. Mitra, Proceedings of the Edinburgh Mathematical Society (2) vol. 4 Part III, p. 111.

6. Earl D. Rainville, Bull. Amer. Math. Soc. vol. 51 (1945) p. 268.

7. N. G. Shabde, Proceedings of the Benares Mathematical Society vol. 7 (1945) pp. 1-2.

8. E. T. Whittaker and G. N. Watson, Modern analysis, 4th ed., 1940.

Government Engineering College, Jabalpur, India 